\title{
Excess hyperpolarizabilities: the irreducible tensor approach
}

\author{
Tadeusz Bancewicz
}

Received: 2 February 2012 / Accepted: 23 February 2012 / Published online: 11 March 2012 C The Author(s) 2012. This article is published with open access at Springerlink.com

\begin{abstract}
The irreducible spherical and Cartesian tensors built of the products of two interaction tensors: the second order tensor resulting from the product of two second order tensors $\mathrm{T}_{\alpha \lambda} \mathrm{T}_{\lambda \beta}$ contracted once with the index $\lambda$, third order tensor $\mathrm{T}_{\alpha \beta \lambda} \mathrm{T}_{\lambda \gamma}$ appearing as a product of the third order interaction tensor $\mathrm{T}_{\alpha \beta \lambda}$ and the second order one $\mathrm{T}_{\lambda \gamma}$ contracted once with the index $\lambda$ and the fourth order product of two second order tensors $\mathrm{T}_{\alpha \beta} \mathrm{T}_{\gamma \delta}$, have been considered. This type of products is encountered, e.g., within the London's dispersive energy formula, inside the secondorder virial coefficients of many physical parameters such as the dielectric constant, the Kerr constant, the induced polarizability and hyperpolarizability of a pair of molecules and in other induced quantities. Our results are applied explicitly to the excess induced first and second pair hyperpolarizability.
\end{abstract}

Keywords Irreducible tensors · First hyperpolarizability ·

Second hyperpolarizability $\cdot$ Collision-induced phenomena

\section{Introduction}

In a gas of moderate density, the magnitude of the moment induced in a molecule is dependent (in addition to the external perturbation) on the internal fields originating in its close neighborhood. The internal fields active in linear as well as nonlinear optics $[1,2]$ phenomena are directly connected to intermolecular interactions. Such fields cause quantum transitions in infrared (IR), Raman and Rayleigh light scattering

\footnotetext{
T. Bancewicz $(\varangle)$

Nonlinear Optics Division, Faculty of Physics, Adam Mickiewicz University, Umultowska 85, 61-614 Poznań, Poland

e-mail: tbancewi@zon12.physd.amu.edu.pl; tbancewi@gmail.com
} 
and in all nonlinear optics effects. In many cases, these transitions are forbidden in the single molecule approximation and lead, e.g., to a collision-induced absorption of radiation by the centrosymmetric microsystems [3], to the depolarized component in the light scattered by systems of atoms and optically isotropic molecules. In the induced transient Raman scattering, internal fields give rise to the anisotropic as well as the isotropic component [4], which for optically isotropic molecules in either case is forbidden in the single molecular scattering. In nonlinear optics, a large part of the second harmonic light scattering measured is attributed to intermolecular interactions $[5,1]$. The collision-induced hyper-Rayleigh light scattering $[6,7]$ originates in internal fields. Moreover, even if a process is permitted in the single molecule approximation, the internal molecular fields cause the phenomenon to deviate considerably from the single molecule approximation.

Within the theory of molecular interactions and induced properties of matter, the interaction tensors $\mathbf{T}^{n}=\underbrace{\nabla \ldots \nabla}_{n-\text { times }}\left(\frac{1}{R}\right)$, for microsystems separated by the distance $R$, play an important role [8-15]. If the described interactions result from the second order of the statistical perturbation theory, the relevant formulas typically contain the product of two such tensors [16]. This type of products enter, e.g., the London's dispersive energy formula (see, for example, [17]) within the second-order virial coefficients of several physical quantities such as the dielectric constant, the Kerr constant, the induced polarizability and hyperpolarizability of a pair of microsystems and other induced quantities. In this study, the irreducible (spherical and Cartesian) form of the fully index-symmetric tensor product of two interaction tensors is considered. Subsequently, we apply our results to interacting pairs of unlike atoms. In particular, our results are applied explicitly to the excess induced first- and second- pair hyperpolarizability.

\section{Second rank tensor $T_{\alpha \lambda} T_{\lambda \beta}$}

The first kind of the product of two interaction tensors often present in molecular physics, e.g., in the second order collision-induced polarizability [16], is of the form $\mathrm{T}_{\alpha \lambda} \mathrm{T}_{\lambda \beta}$. We denote this tensor as ${ }^{(2)} \Delta \mathbf{A}$. In the irreducible spherical tensor language this product, in the self-explanatory notation, reads

$$
{ }^{(2)} \Delta \mathrm{A}_{k \rho}=\left\{\left(\boldsymbol{\mu}_{1} \otimes \boldsymbol{\eta}_{1}\right)^{(k)} \otimes\left(\boldsymbol{\mu}_{1} \odot \boldsymbol{\eta}_{1}\right)\right\}_{k \rho} .
$$

where $\otimes$ denotes the tensor product and $\odot$ stands for the scalar product and we connect the $\mu_{1}$ vector with the first tensor of $\mathrm{T}_{\alpha \lambda} \mathrm{T}_{\lambda \beta}$ and $\eta_{1}$ with the second tensor.

Using recouping procedures [18] we obtain

$$
{ }^{(2)} \Delta \mathrm{A}_{k \rho}=\left(\mathrm{T}_{\alpha \lambda} \mathrm{T}_{\lambda \beta}\right)_{k \rho}=-5(-1)^{k}\left\{\begin{array}{lll}
2 & 2 & k \\
1 & 1 & 1
\end{array}\right\}\left\{\mathbf{T}^{(2)} \otimes \mathbf{T}^{(2)}\right\}_{k \rho},
$$

where $\{\ldots\}$ is the 6-j Wigner symbol, and $\mathbf{T}^{(2)}$ is the spherical second rank interaction tensor. Note that for this tensor there is only one coupling scheme possible. For the isotropic and the anisotropic component of $\Delta \mathrm{A}_{k \rho}$, we obtain 


$$
\text { (2) } \Delta \mathrm{A}_{00}=-2 \sqrt{3} R^{-6} \text {, }
$$

or in the Cartesian notation for the mean value of the tensor ${ }^{(2)} \Delta \mathbf{A}$

$$
\overline{(2) \Delta \mathbf{A}}=\frac{{ }^{(2)} \Delta \mathbf{A}_{\rho \rho}}{3}=2 R^{-6}
$$

and

$$
{ }^{(2)} \Delta \mathrm{A}_{20}=3 \sqrt{\frac{2}{3}} R^{-6},
$$

or in the Cartesian notation for its anisotropy

$$
{ }^{(2)} \Delta \mathbf{A}={ }^{(2)} \Delta \mathrm{A}_{z z}-{ }^{(2)} \Delta \mathbf{A}_{x x}=3 R^{-6} \text {. }
$$
(2) $\Delta \mathbf{A}$

It is interesting and useful to calculate the Cartesian irreducible $z z$ components of

$$
\begin{aligned}
{ }^{(2)} \Delta \mathrm{A}_{z z}^{(0)} & =2 R^{-6} \\
{ }^{(2)} \Delta \mathrm{A}_{z z}^{(2)} & =2 R^{-6} .
\end{aligned}
$$

Note that the $T_{\alpha \lambda} T_{\lambda \beta}$ tensor has already been discussed extensively in Ref. [16]. The above results give us immediately the well known formulas for the trace of the polarizability of the diatom

$$
\alpha(R)=\frac{4 \alpha^{3}}{R^{6}}
$$

and its anisotropy

$$
\gamma(R)=\frac{6 \alpha^{3}}{R^{6}},
$$

with $\alpha$-the intrinsic polarizability of an atom.

\section{Third rank tensor $\mathbf{T}_{\alpha \beta \lambda} \mathbf{T}_{\lambda \gamma}$}

We consider the product $T_{\alpha \beta \lambda} T_{\lambda \gamma}$ of the second and third rank interaction tensors contracted once with the index $\lambda$. Moreover, we are interested in this tensor symmetrized over all its indexes $\alpha \beta \gamma$. We denote this symmetrized tensor by ${ }^{(s)} \Delta \mathrm{F}_{\alpha \beta \gamma}$

$$
{ }^{(s)} \Delta \mathrm{F}_{\alpha \beta \gamma}=\frac{1}{3 !} S_{\alpha \beta \gamma}\left(\mathrm{T}_{\alpha \beta \lambda} \mathrm{T}_{\lambda \gamma}\right),
$$

where $S_{\alpha \beta \gamma}$ stands for the summation over all permutations of the set $\alpha \beta \gamma$. 


\subsection{Irreducible spherical considerations}

Let us consider first the tensor $\mathrm{B}_{\alpha \beta \gamma}=\mathrm{T}_{\alpha \beta \lambda} \mathrm{T}_{\lambda \gamma}$. In the spherical notation we rewrite this tensor in the following way

$$
\mathrm{B}_{k \rho}=\left\{\left(\boldsymbol{\mu}_{1} \otimes \boldsymbol{\mu}_{1}\right)^{(2)} \otimes\left(\boldsymbol{\mu}_{1} \odot \boldsymbol{\eta}_{1}\right) \boldsymbol{\eta}_{1}\right\}_{k \rho} .
$$

Using decoupling procedures [18] we find

$$
\mathrm{B}_{k \rho}[(31) a 1]=(-1)^{k+a} \sqrt{35}\left\{\begin{array}{lll}
3 & 2 & k \\
1 & a & 1
\end{array}\right\}\left\{\mathbf{T}^{(3)} \otimes \mathbf{T}^{(2)}\right\}_{k \rho},
$$

or explicitly

$$
\begin{gathered}
\mathrm{B}_{10}[(31), 21]=18 \sqrt{\frac{3}{5}} R^{-7}, \\
\mathrm{~B}_{30}[(31), 21]=-12 \sqrt{\frac{2}{5}} R^{-7} .
\end{gathered}
$$

In Eq. (13) first we couple the components (((11)21)3) of the $T_{\alpha \beta \lambda}$ tensor and subsequently the components of $\mathrm{T}_{\lambda \gamma}$. Let us remind that the interaction tensors are irreducible and traceless. To obtain the index-symmetric third rank tensor ${ }^{(s)} \Delta \mathrm{F}_{\alpha \beta \gamma}$ from Eq. (13), the genealogical coefficients are necessary. They are given in Table 1. For the notation $\lambda_{\tau}^{3 j}$ of the genealogical coefficients see [19,20]. In Eq. (13), the set of the last two indexes (a1) forms the seniority index $\tau$ for this tensor.

To relate the irreducible spherical tensor components to the Cartesian components, the transformation coefficients are needed. Symmetry considerations show that for the $\mathrm{D}_{\infty} v$ point group and for the third order index-symmetric tensor $\mathbf{D}$ only two linearly independent components $\mathrm{D}_{x x z}$ and $\mathrm{D}_{z z z}$ exist. In the irreducible spherical notation they correspond to $D_{10}$ and $D_{30}$. The transformation coefficients between these two sets are collected in Table 2. In our considerations we use the well known Condon and Shorthey phase convention [20].

Table 1 The genealogical coefficients $\lambda_{\tau}^{3, j}$ for an index-symmetric third rank tensor

\begin{tabular}{lll}
\hline & $j=1$ & $j=3$ \\
\hline$\lambda_{01}^{3 j}$ & $\frac{\sqrt{5}}{3}$ & 0 \\
$\lambda_{21}^{3 j}$ & $\frac{2}{3}$ & 1 \\
\hline
\end{tabular}


Table 2 The

Cartesian-spherical transformation coefficients for the third rank symmetric tensor D

\begin{tabular}{lcc}
\hline & $\mathrm{D}_{x x z}$ & $\mathrm{D}_{z z z}$ \\
\hline $\mathrm{D}_{10}$ & $-\frac{1}{\sqrt{15}}$ & $-\sqrt{\frac{3}{5}}$ \\
$\mathrm{D}_{30}$ & $-\frac{1}{\sqrt{10}}$ & $\sqrt{\frac{2}{5}}$ \\
\hline
\end{tabular}

\subsection{Irreducible Cartesian tensors}

Concurrently, we consider the irreducible parts of the ${ }^{(s)} \Delta \mathrm{F}_{\alpha \beta \gamma}$ tensor in the Cartesian form. In general, the totally index-symmetric third rank tensor $D$ has only odd order irreducible components of the form [20-22]:

(a) the first order component

$$
\mathrm{D}_{\alpha \beta \gamma}^{(1)}=\frac{1}{5}\left(\delta_{\alpha \beta} \mathrm{D}_{\rho \rho \gamma}+\delta_{\alpha \gamma} \mathrm{D}_{\rho \rho \beta}+\delta_{\beta \gamma} \mathrm{D}_{\rho \rho \alpha}\right)
$$

(b) the third order component

$$
\mathrm{D}_{\alpha \beta \gamma}^{(3)}=\mathrm{D}_{\alpha \beta \gamma}-\mathrm{D}_{\alpha \beta \gamma}^{(1)} \text {. }
$$

Applying the above formula to the ${ }^{(s)} \Delta \mathrm{F}_{\alpha \beta \gamma}$ tensor we obtain the following results:

$$
{ }^{(s)} \Delta \mathrm{F}_{\alpha \rho \rho}=-12 \hat{R}_{\alpha} R^{-7}
$$

and

$$
{ }^{(s)} \Delta \mathrm{F}_{\alpha \beta \gamma}^{(1)}=-\frac{12}{5}\left(\hat{R}_{\alpha} \delta_{\beta \gamma}+\hat{R}_{\beta} \delta_{\alpha \gamma}+\hat{R}_{\gamma} \delta_{\alpha \beta}\right) R^{-7} .
$$

The relation (19) yields

$$
{ }^{(s)} \Delta \mathrm{F}_{z z z}^{(1)}=-\frac{36}{5} R^{-7}
$$

and according to Eq. (17) we have

$$
{ }^{(s)} \Delta \mathrm{F}_{z z z}^{(3)}=\left(-12+\frac{36}{5}\right) R^{-7}=-\frac{24}{5} R^{-7}
$$

The results are given in Table 5. We note a good agreement between the irreducible Cartesian and irreducible spherical tensors. 


\section{Fourth rank tensor $T_{\alpha \beta} T_{\gamma \delta}$}

Now we consider the index symmetric fourth rank product of two interaction tensors. We denote this tensor as ${ }^{(s)} \Delta \mathrm{E}_{\alpha \beta \gamma \delta}$

$$
{ }^{(s)} \Delta \mathrm{E}_{\alpha \beta \gamma \delta}=\frac{1}{4 !} S_{\alpha \beta \gamma \delta}\left(\mathrm{T}_{\alpha \beta} \mathrm{T}_{\gamma \delta}\right)
$$

\subsection{Irreducible spherical approach}

First we consider the fourth rank tensor $\mathrm{C}_{\alpha \beta \gamma \delta}=\mathrm{T}_{\alpha \beta} \mathrm{T}_{\gamma \delta}$. In the irreducible spherical notation this tensor reads:

$$
{ }^{(s)} \mathrm{C}_{k \rho}=\left\{\left(\boldsymbol{\mu}_{1} \otimes \boldsymbol{\mu}_{1}\right)^{(2)} \otimes\left(\boldsymbol{\eta}_{1} \times \boldsymbol{\eta}_{1}\right)^{(2)}\right\}_{k \rho} .
$$

Using recouping procedures we rewrite Eq. (23) as follows:

$$
\begin{gathered}
{ }^{(s)} \mathrm{C}_{k \rho}[(21) a 1]=\left\{\left(\mathbf{T}^{(2)} \otimes \boldsymbol{\mu}_{1}\right)^{(a)} \otimes \boldsymbol{\mu}_{1}\right\}_{k \rho} \\
=(-1)^{k} \sqrt{5(2 a+1)}\left\{\begin{array}{lll}
2 & 1 & a \\
1 & k & 2
\end{array}\right\}\left\{\mathbf{T}^{(2)} \otimes \mathbf{T}^{(2)}\right\}_{k \rho} .
\end{gathered}
$$

The coupling scheme is visible in the first line of Eq. (24). This equation makes it possible to obtain the set of the subsequent non-zero components of the index-symmetric part of the tensor ${ }^{(2)} \Delta \mathbf{E}$. They are of the form:

$$
\begin{aligned}
& \mathrm{C}_{00}[(21) 11]=\left\{\mathbf{T}^{(2)} \otimes \mathbf{T}^{(2)}\right\}_{00}, \\
& \mathrm{C}_{20}[(21) 11]=\frac{\sqrt{\frac{7}{5}}}{2}\left\{\mathbf{T}^{(2)} \otimes \mathbf{T}^{(2)}\right\}_{20}, \\
& \mathrm{C}_{20}((21) 31]=\frac{1}{\sqrt{15}}\left\{\mathbf{T}^{(2)} \otimes \mathbf{T}^{(2)}\right\}_{20}, \\
& \mathrm{C}_{40}((21) 31]=\left\{\mathbf{T}^{(2)} \otimes \mathbf{T}^{(2)}\right\}_{40} .
\end{aligned}
$$

For these components the coupling steps are clearly visible. The seniority index of the ordinary fourth rank tensor composed by four dipoles $\mathbf{D}_{k \rho}\left[\left((11) j_{2} 1\right) j_{3} 1\right]$ is given by the set $\tau=\left(j_{2} j_{3}\right)$. The interaction tensors are irreducible and traceless, then in our tensor $\mathrm{C}, j_{2}=2$ We write our tensor $\mathrm{C}$ in the form $\mathrm{C}_{k \rho}\left[(21) j_{3} 1\right]$. Then seniority index of our tensor $\mathbf{C}$ reads, $\tau=\left(2 j_{3}\right)$. Again, the genealogical coefficients are necessary in order to obtain the index symmetric part of the tensor $\mathrm{C}_{\alpha \beta \gamma \delta}$. The genealogical coefficients for a fourth-rank totally index-symmetric tensor are collected in Table 3. 
Table 3 The genealogical coefficients for a fourth-rank totally index-symmetric tensor

\begin{tabular}{llll}
\hline & $j=0$ & $j=2$ & $j=4$ \\
\hline$\lambda_{01}^{4 j}$ & $\frac{\sqrt{5}}{3}$ & $\frac{\sqrt{14}}{3}$ & 0 \\
$\lambda_{21}^{4 j}$ & $\frac{2}{3}$ & $\frac{\sqrt{14 / 5}}{3}$ & 0 \\
$\lambda_{23}^{4 j}$ & 0 & $\sqrt{\frac{3}{10}}$ & 1 \\
\hline
\end{tabular}

\begin{tabular}{llcc}
\hline & $\mathrm{D}_{x x x x}$ & $\mathrm{D}_{x x z z}$ & $\mathrm{D}_{z z z z}$ \\
\hline $\mathrm{D}_{00}$ & $\frac{1}{\sqrt{5}}$ & $\frac{1}{3 \sqrt{5}}$ & $\frac{1}{\sqrt{5}}$ \\
$\mathrm{D}_{20}$ & $\frac{1}{\sqrt{7}}$ & $-\frac{1}{6 \sqrt{7}}$ & $-\frac{2}{\sqrt{7}}$ \\
$\mathrm{D}_{40}$ & $\frac{3}{2 \sqrt{70}}$ & $-\frac{2}{\sqrt{70}}$ & $\frac{4}{\sqrt{70}}$ \\
\hline
\end{tabular}

Table 4 The

Cartesian-spherical

transformation coefficients for index-symmetric fourth-rank tensor $\mathcal{D}$

The results of Eq. (25) and Table 3 allow us to calculate the components of the ${ }^{(s)} \Delta \mathbf{E}$ tensor

$$
\begin{aligned}
& { }^{(s)} \Delta \mathrm{E}_{00}=\frac{2}{3} \frac{6}{\sqrt{5}} R^{-6}=\frac{4}{\sqrt{5}} R^{-6}, \\
& { }^{(s)} \Delta \mathrm{E}_{20}=\left(\frac{\sqrt{\frac{14}{5}}}{3} \frac{\sqrt{\frac{7}{5}}}{2}+\sqrt{\frac{3}{10}} \frac{1}{\sqrt{15}}\right)\left(-\sqrt{\frac{2}{7}}\right) 6 R^{-6}=-\frac{4}{\sqrt{7}} R^{-6}, \\
& { }^{(s)} \Delta \mathrm{E}_{40}=6 \sqrt{\frac{3}{35}} R^{-6} .
\end{aligned}
$$

In Eq. (26), the first factor stands for the respective genealogical coefficient and the second one results from Eq. (25). In order to relate the irreducible spherical components $\Delta \mathrm{E}_{j 0}$ to the Cartesian ones, we use the transformation coefficients collected in Table 4. Symmetry considerations show that for the $\mathrm{C}_{\infty} v$ and $\mathrm{D}_{\infty} v$ point groups and for the fourth order fully index-symmetric tensor $\mathbf{D}$ there exist only three linearly independent components $\mathrm{D}_{x x x x}, \mathrm{D}_{x x z z}$ and $\mathrm{D}_{z z z z}$. In the irreducible spherical notation they correspond to $D_{00}, D_{20}$ and $D_{40}$.

\subsection{Irreducible Cartesian picture}

Concurrently, the totally index-symmetric fourth rank tensor D has only even order irreducible components of the form [20,22]:

(a) the zero order component

$$
\mathrm{D}_{\alpha \beta \gamma \delta}^{(0)}=\frac{1}{15}\left(\delta_{\alpha \beta} \delta_{\gamma \delta}+\delta_{\alpha \gamma} \delta_{\beta \delta}+\delta_{\alpha \delta} \delta_{\beta \gamma}\right) \mathrm{D}_{\rho \rho \sigma \sigma}
$$


(b) the second order component

$$
\begin{aligned}
\mathrm{D}_{\alpha \beta \gamma \delta}^{(2)}= & \frac{1}{7}\left(\delta_{\alpha \beta} \mathrm{D}_{\rho \rho \gamma \delta}+\delta_{\beta \delta} \mathrm{D}_{\rho \rho \alpha \gamma}+\delta_{\gamma \delta} \mathrm{D}_{\rho \rho \alpha \beta}\right. \\
& \left.+\delta_{\alpha \gamma} \mathrm{D}_{\rho \rho \beta \delta}+\delta_{\alpha \delta} \mathrm{D}_{\rho \rho \beta \gamma}+\delta_{\beta \gamma} \mathrm{D}_{\rho \rho \alpha \delta}\right)-\frac{10}{7} \mathrm{D}_{\alpha \beta \gamma \delta}^{(0)}
\end{aligned}
$$

(c) the fourth order component

$$
\mathrm{D}_{\alpha \beta \gamma \delta}^{(4)}=\mathrm{D}_{\alpha \beta \gamma \delta}-\mathrm{D}_{\alpha \beta \gamma \delta}^{(0)}-\mathrm{D}_{\alpha \beta \gamma \delta}^{(2)}
$$

Let us apply the formulas (27-29) to the ${ }^{(s)} \Delta \mathbf{E}$ tensor given by Eq. (22). The ${ }^{(s)} \Delta \mathbf{E}$ tensor contracted with two indexes gives

$$
{ }^{(s)} \Delta \mathrm{E}_{\rho \rho \gamma \delta}=\frac{2}{3}\left(\mathbf{T}^{(2)} \cdot \mathbf{T}^{(2)}\right)_{\gamma \delta}
$$

Contracting further on we obtain

$$
{ }^{(s)} \Delta \mathrm{E}_{\rho \rho \sigma \sigma}=4 R^{-6} \text {. }
$$

Using Eqs. (27-29) and the above result we obtain

$$
\begin{aligned}
{ }^{(s)} \Delta \mathrm{E}_{z z z z}^{(0)} & =\frac{4}{5} R^{-6}, \\
{ }^{(s)} \Delta \mathrm{E}_{z z z z}^{(2)} & =\frac{8}{7} R^{-6}, \\
{ }^{(s)} \Delta \mathrm{E}_{z z z z}^{(4)} & =\frac{72}{35} R^{-6} .
\end{aligned}
$$

\section{Second order correction to the redundant first and second hyperpolarizability tensor}

With the aim of presenting applications of our method we calculate the excess second order correction to the induced first- and second- order hyperpolarizability. It has been shown by Buckingham [14], Hunt [23] and Li et al. [13], note also [15], that for a pair of atoms $\mathrm{A}$ and $\mathrm{B}$ the second order correction to the induced first-hyperpolarizability tensor $\Delta \beta$ is given by the following formula

$$
{ }^{(2)} \Delta \beta_{\alpha \beta \gamma}=-\frac{1}{2} S_{\alpha \beta \gamma}\left(\mathrm{T}_{\alpha \gamma \lambda} \mathrm{T}_{\lambda \beta}\right) B_{A} \alpha_{A} \alpha_{B}-\frac{1}{4} S_{\alpha \beta \gamma}\left(\mathrm{T}_{\alpha \gamma \lambda} \mathrm{T}_{\lambda \beta}\right) B_{A} \alpha_{A}^{2} \text {. }
$$


Table 5 Results for the excess first-hyperpolarizability $\Delta \beta$

\begin{tabular}{|c|c|c|c|}
\hline \multirow[t]{4}{*}{${ }^{(s)} \Delta \mathrm{F}_{\alpha \beta \gamma}$} & $\begin{array}{l}{ }^{(s)} \Delta \mathrm{F}_{10} \\
12 \sqrt{\frac{3}{5}} R^{-7}\end{array}$ & $\begin{array}{l}{ }^{(s)} \Delta \mathrm{F}_{30} \\
-12 \sqrt{\frac{2}{5}} R^{-7}\end{array}$ & \\
\hline & $\begin{array}{l}\Delta \mathrm{F}_{z z z}^{(1)} \\
-\frac{36}{5} R^{-7}\end{array}$ & $\begin{array}{l}\Delta \mathrm{F}_{z z z}^{(3)} \\
-\frac{24}{5} R^{-7}\end{array}$ & $\begin{array}{l}\Delta \mathrm{F}_{z z z} \\
-12 R^{-7}\end{array}$ \\
\hline & $\begin{array}{l}\Delta \beta_{z z z}^{(1)} \\
\frac{108}{5} B_{A} \alpha_{A} \alpha_{B} R^{-7}\end{array}$ & $\begin{array}{l}\Delta \beta_{z z z}^{(3)} \\
\frac{72}{5} B_{A} \alpha_{A} \alpha_{B} R^{-7}\end{array}$ & $\begin{array}{l}\Delta \beta_{z z z} \\
36 B_{A} \alpha_{A} \alpha_{B} R^{-7}\end{array}$ \\
\hline & $\begin{array}{l}\Delta \beta_{z z z}^{(1)} \\
\frac{54}{5} B_{A} \alpha_{B}^{2} R^{-7}\end{array}$ & $\begin{array}{l}\Delta \beta_{z z z}^{(3)} \\
\frac{36}{5} B_{A} \alpha_{B}^{2} R^{-7}\end{array}$ & $\begin{array}{l}\Delta \beta_{z z z} \\
18 B_{A} \alpha_{B}^{2} R^{-7}\end{array}$ \\
\hline
\end{tabular}

For the second order correction to the redundant induced second hyperpolarizability $\Delta \gamma$ we have $[14,13]$ :

$$
\begin{aligned}
\left(\gamma_{A} \alpha_{B}^{2}\right){ }^{(2)} \Delta \gamma_{\alpha \beta \gamma \delta}= & \frac{1}{12} S_{\alpha \beta \gamma \delta}\left(\delta_{\alpha \beta} \mathrm{T}_{\lambda \gamma} \mathrm{T}_{\lambda \delta}+\mathrm{T}_{\alpha \gamma} \mathrm{T}_{\beta \delta}+\mathrm{T}_{\alpha \delta} \mathrm{T}_{\beta \gamma}\right) \gamma_{A} \alpha_{B}^{2}, \\
\left(\gamma_{A} \alpha_{A} \alpha_{B}\right){ }^{(2)} \Delta \gamma_{\alpha \beta \gamma \delta}= & \frac{1}{18} S_{\alpha \beta \gamma \delta}\left(\delta_{\gamma \delta} \mathrm{T}_{\alpha \lambda} \mathrm{T}_{\lambda \beta}+\delta_{\beta \delta} \mathrm{T}_{\alpha \lambda} \mathrm{T}_{\lambda \gamma}+\delta_{\beta \gamma} \mathrm{T}_{\alpha \lambda} \mathrm{T}_{\lambda \delta}\right) \\
& \times \gamma_{A} \alpha_{A} \alpha_{B},
\end{aligned}
$$

where $B_{i}, \alpha_{i}$ and $\gamma_{i}$ denote the dipole ${ }^{2}$-quadrupole hyperpolarizability, polarizability and second-hyperpolarizability of an atom $i$, respectively.

Using the hereinbefore mentioned methods, we calculate the irreducible spherical components ${ }^{(s)} \Delta \mathrm{F}_{10},{ }^{(s)} \Delta \mathrm{F}_{30}$. The results are given in Table 5. In the latter Table the Cartesian $z z z$ components of $\Delta \mathbf{F}$ calculated using the irreducible Cartesian tensor approach are given as well. As an example, let us calculate the dipolar part ${ }_{\left(B_{A} \alpha_{B}^{2}\right)} \Delta \beta_{z z z}^{(1)}$ of the first hyperpolarizability. We have

$$
{ }_{\left(B_{A} \alpha_{B}^{2}\right)} \Delta \beta_{z z z}^{(1)}=\frac{3}{2} \sqrt{\frac{3}{5}} 12 \sqrt{\frac{3}{5}} B_{A} \alpha_{B}^{2} R^{-7}=\frac{54}{5} B_{A} \alpha_{B}^{2} R^{-7} .
$$

The first coefficient in Eq. (36) results from the formula (33) if all permutations are considered, the second coefficient comes from Table 2 and the third one-from Table 5 .

Similar arguments apply to the ${ }^{(s)} \Delta \mathbf{E}$ tensor and the second-hyperpolarizability tensor. The ${ }^{(s)} \Delta E_{\alpha \beta \gamma \delta}$ tensor components ${ }^{(s)} \Delta \mathrm{E}_{00},{ }^{(s)} \Delta \mathrm{E}_{20}$ and ${ }^{(s)} \Delta \mathrm{E}_{40}$ are given in Table 6. In the latter Table the Cartesian $z z z z$ components of ${ }^{(s)} \Delta \mathbf{E}$ calculated using the irreducible Cartesian tensor approach are given as well. Note again a good agreement between these results. Let us present an example of using the data of Table 6 . Now, we calculate the ${ }^{(2)} \Delta \gamma_{z z z z}^{(0)}$ contribution from the components given in Table 6:

$$
{ }_{\left(\gamma_{A} \alpha_{A} \alpha_{B}\right)} \Delta \gamma_{z z z z}^{(0)}=4 \cdot 2 \gamma_{A} \alpha_{A} \alpha_{B} R^{-6}=8 \gamma_{A} \alpha_{A} \alpha_{B} R^{-6},
$$


Table 6 Results for the excess second-hyperpolarizability $\Delta \gamma$

\begin{tabular}{lllll}
\hline${ }^{(s)} \Delta E_{\alpha \beta \gamma \delta}$ & ${ }^{(s)} \Delta \mathrm{E}_{00}$ & ${ }^{(s)} \Delta \mathrm{E}_{20}$ & ${ }^{(s)} \Delta \mathrm{E}_{40}$ & \\
& $\frac{4}{\sqrt{5}} R^{-6}$ & $-\frac{4}{\sqrt{7}} R^{-6}$ & $3 \sqrt{\frac{2}{35}} R^{-6}$ & \\
& ${ }^{(s)} \Delta \mathrm{E}_{z z z z}^{(0)}$ & ${ }^{(s)} \Delta \mathrm{E}_{z z z z}^{(2)}$ & ${ }^{(s)} \Delta \mathrm{E}_{z z z z}^{(4)}$ & ${ }^{(s)} \Delta \mathrm{E}_{z z z z}$ \\
& $\frac{4}{5} R^{-6}$ & $\frac{8}{7} R^{-6}$ & $\frac{72}{35} R^{-6}$ & $4 R^{-6}$ \\
${ }^{(2)} \Delta \mathrm{A}_{\alpha \beta}$ & ${ }^{(2)} \Delta \mathrm{A}_{00}$ & $(2) \Delta \mathrm{A}_{20}$ & ${ }^{(2)} \Delta \mathrm{A}_{40}$ & \\
& $-2 \sqrt{3} R^{-6}$ & $3 \sqrt{\frac{2}{3}} R^{-6}$ & 0 & \\
& $(2) \Delta \mathrm{A}_{z z}^{(0)}$ & $(2) \Delta \mathrm{A}_{z z}^{(2)}$ & ${ }^{(2)} \Delta \mathrm{A}_{z z}^{(4)}$ & $(2) \Delta \mathrm{A}_{z z}$ \\
& $2 R^{-6}$ & $2 R^{-6}$ & 0 & $4 R^{-6}$ \\
& $(2) \Delta \gamma_{z z z z}^{(0)}$ & $(2) \Delta \gamma_{z z z z}^{(2)}$ & ${ }^{(2)} \Delta \gamma_{z z z z}^{(4)}$ & $(2) \Delta \gamma_{z z z z}$ \\
${ }^{(2)} \Delta \gamma_{\alpha \beta \gamma \delta}$ & $8 \gamma_{A} \alpha_{A} \alpha_{B} R^{-6}$ & $8 \gamma_{A} \alpha_{A} \alpha_{B} R^{-6}$ & 0 & $16 \gamma_{A} \alpha_{A} \alpha_{B} R^{-6}$ \\
& $(2) \Delta \gamma_{z z z z}^{(0)}$ & $(2) \Delta \gamma_{z z z z}^{(2)}$ & ${ }^{(2)} \Delta \gamma_{z z z z}^{(4)}$ & $(s) \Delta \gamma_{z z z z}$ \\
& $\frac{36}{5} \gamma_{A} \alpha_{B}^{2} R^{-6}$ & $\frac{60}{7} \gamma_{A} \alpha_{B}^{2} R^{-6}$ & $\frac{288}{35} \gamma_{A} \alpha_{B}^{2} R^{-6}$ & $24 \gamma_{A} \alpha_{B}^{2} R^{-6}$ \\
\hline
\end{tabular}

where the first factor in Eq. (37) originates from all possible permutations of Eq. (35). Next, we use the results of Eq. (7).

Additionally, for $\left(\gamma_{A} \alpha_{B}^{2}\right) \Delta \gamma_{z z z z}^{(0)}$ we obtain

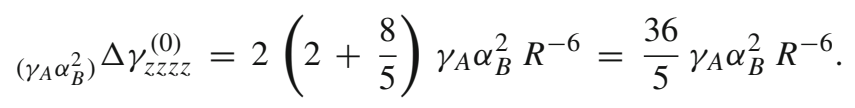

The factor 2 in Eq. (38) results from all possible permutations in Eq. (35). On the other hand, the first term in the parenthesis in Eq. (35) (according to Eq. (7)) yields $2 \gamma_{A} \alpha_{B}^{2} R^{-6}$. The last two terms result from the value ${ }^{(s)} \Delta \mathrm{E}_{00}=\frac{4}{\sqrt{5}} R^{-6}$ in Table 6 and the respective genealogical coefficient $\left(\frac{1}{\sqrt{5}}\right)$ in Table 4 leads to the result $\frac{8}{5}$. In a similar way, we obtain

$$
\left(\gamma_{A} \alpha_{B}^{2}\right) \Delta \gamma_{z z z z}^{(2)}=2\left(2+\frac{16}{7}\right) \gamma_{A} \alpha_{B}^{2} R^{-6}=\frac{60}{7} \gamma_{A} \alpha_{B}^{2} R^{-6} .
$$

The last formula (40) needs only the respective Clebsch-Gordon coefficient, the transformation coefficient and the permutation factor:

$$
{ }_{\left(\gamma_{A} \alpha_{B}^{2}\right)} \Delta \gamma_{z z z z}^{(4)}=\frac{4}{\sqrt{70}} 72 \sqrt{\frac{2}{35}} \gamma_{A} \alpha_{B}^{2} R^{-6}=\frac{288}{35} \gamma_{A} \alpha_{B}^{2} R^{-6}
$$




\section{Conclusion}

The irreducible spherical and Cartesian tensors built of the products of two interaction tensors: the second order tensor resulting from the product of two second order tensors $\mathrm{T}_{\alpha \lambda} \mathrm{T}_{\lambda \beta}$ contracted once with the index $\lambda$, third order tensor $\mathrm{T}_{\alpha \beta \lambda} \mathrm{T}_{\lambda \gamma}$ appearing as a product of the third order interaction tensor $T_{\alpha \beta \lambda}$ and the second order one $\mathrm{T}_{\lambda \gamma}$ contracted once with the index $\lambda$ as well as the fourth order product of two second order tensors $\mathrm{T}_{\alpha \beta} \mathrm{T}_{\gamma \delta}$ have been considered The results have been applied to the excess first and second collision-induced hyperpolarizability tensor of a pair of dissimilar atoms. Excellent agreement with the results found by Buckingham et al. [14] as well as the results by Hunt [23] and $\mathrm{Li}$ et al. [12,13] has been found. It is interesting to note that the irreducible Cartesian components can be obtained directly from Cartesian considerations (see Sects. 3.2, 4.2) or through the irreducible spherical components and the respective transformation coefficients (Tables 2, 4). A good agreement has been found.

Acknowledgments This paper has been supported by the research project Nb. N N202 069939 sponsored by The Government of Poland. I would like to thank very much George Maroulis for many illuminating discussions.

Open Access This article is distributed under the terms of the Creative Commons Attribution License which permits any use, distribution, and reproduction in any medium, provided the original author(s) and the source are credited.

\section{References}

1. S. Kielich, Prog. Opt. XX, 155 (1983)

2. G. Maroulis, T. Bancewicz, B. Champagne, A.D.Buckingham (eds.), Atomic and Molecular Nonlinear Optics: Theory, Experiment and Computation. A Homage to the Pioneering Work of Stanisaw Kielich (1925-1993)(IOS Press, Amsterdam, 2011)

3. L. Frommhold, Collision-induced Absorption in Gases (Cambridge University Press, Cambridge, 1993)

4. T. Bancewicz, Y. Le Duff, J.-L. Godet, Modern Nonlinear Optics, Part 1, Advances in Chemical Physics, Vol. 119, 2nd edn, ed. by M. Evans (Wiley, New York, 2000), pp. 89-126

5. P. Kaatz, D.P. Shelton, Mol. Phys. 88, 683 (1996)

6. W. Głaz, T. Bancewicz, J.-L. Godet, J. Chem. Phys. 122, 224323 (2005)

7. T. Bancewicz, W. Głaz, J.-L. Godet, G. Maroulis, J. Chem. Phys. 129, 124306 (2008)

8. S. Kielich, Nonlinear Molecular Optics (Nauka, Moscow, 1981)

9. G. Maroulis, J. Phys. Chem. A 104, 4772 (2000)

10. T. Bancewicz, G. Maroulis, Phys. Rev. A 79, 042704 (2009)

11. T. Bancewicz, G. Maroulis, Chem. Phys. Lett. 498, 349 (2010)

12. X. Li, K.L.C. Hunt, J. Chem. Phys. 100, 7875 (1994)

13. X. Li, K.L.C. Hunt, J. Pipin, D.M. Bishop, J. Chem. Phys. 105, 10954 (1996)

14. A.D. Buckingham, E.P. Concannon, I.D. Hands, J. Phys. Chem. 98, 10455 (1994)

15. T. Bancewicz, J. Chem. Phys. 111, 7440 (1999)

16. T. Bancewicz, Mol. Phys. 50, 173 (1983)

17. C.G. Gray, K.E. Gubbins, Theory of Molecular Fluids. Vol. 1: Fundamentals (Clarendon Press, Oxford, 1984)

18. D.A. Varshalovich, A.N. Moskaliev, V.K. Khersonskii, Quantum Theory of Angular Momentum (World Scientific, Singapore, 1988)

19. P.D. Maker, Phys. Rev. A 1, 923 (1970)

20. J. Jerphagnon, D. Chemla, R. Bonneville, Adv. Phys. 27, 609 (1978) 
21. A.J. Stone, Mol. Phys. 29, 1461 (1975)

22. D.L. Andrews, W.A. Ghoul, J. Chem. Phys. 75, 530 (1981)

23. K.L.C. Hunt, Chem. Phys. Lett. 70, 336 (1980) 